23

\title{
Акустооптический гиперспектральный модуль для гистологического исследования микрообъектов
}

\author{
() О.В. Польщикова ${ }^{1}$, А.С. Мачихин ${ }^{1,2}$, А.Г. Рамазанова ${ }^{1}$, И.А. Братченко ${ }^{3}$, В.Э. Пожар ${ }^{1}$, \\ И.В. Данилычева ${ }^{4,5}$, О.Р. Катунина ${ }^{6}$, М.В. Данилычев ${ }^{7}$ \\ ${ }^{1}$ Научно-технологический центр уникального приборостроения РАН, \\ 117342 Москва, Россия \\ ${ }^{2}$ Национальный исследовательский университет „МЭИ“, \\ 111250 Москва, Россия \\ ${ }^{3}$ Самарский национальный исследовательский университет им. академика С.П. Королева, \\ 443086 Самара, Россия \\ ${ }^{4}$ Государственный научный центр „Институт иммунологии“ Федерального медико-биологического агентства, \\ 115478 Москва, Россия \\ ${ }^{5}$ Московский государственный медико-стоматологический университет им. А.И. Евдокимова, \\ 127473 Москва, Россия \\ ${ }^{6}$ Государственный научный центр Российской Федерации - \\ Федеральный медицинский биофизический центр им. А.И. Бурназяна, \\ 123098 Москва, Россия \\ ${ }^{7}$ Институт радиотехники и электроники им. В.А. Котельникова РАН, \\ 125009 Москва, Россия \\ e-mail: polschikova@gmail.com
}

Поступила в редакцию 31.07.2018 г.

В окончательной редакции 06.10.2018 г.

Рассмотрена задача создания съемного видеоспектрометрического модуля, встраиваемого в оптическую систему микроскопов, на основе перестраиваемого акустооптического фильтра для гиперспектрального анализа микрообъектов. Для этого осуществлено светоэнергетическое сопряжение фильтра с микроскопом. Проведен расчет сопрягающей оптической системы, обеспечивающей равенство полей зрения широкополосного и спектрального каналов. Описан макет разработанного прибора. Приведены примеры регистрации изображений тест-объектов и гистологических срезов и результаты вычисления спектральных характеристик различных тканей кожи. Разработанный видеоспектрометрический модуль существенно расширяет функциональные возможности микроскопов и может найти широкое применение для различных задач биомедицинской диагностики.

DOI: 10.21883/OS.2019.02.47211.227-18

\section{Введение}

Гиперспектральный анализ находит широкое применение в различных областях науки и техники. Все более актуальной она становится и в задачах медицинской диагностики, в частности, для микроскопического исследования тканей и клеток в белом свете и в режиме флуоресценции, которое обычно выполняется опытными специалистами-патоморфологами. Развитие гиперспектральных методов биомедицинских исследований связано со стремлением автоматизировать процессы диагностики, повысить их точность, исключить человеческий фактор. Их главным преимуществом является получение количественной информации о таких оптических свойствах биологических тканей, как спектры поглощения, рассеяния и флуоресценции, которые зависят от состояния тканей, стадии заболевания и других факторов.

Гиперспектральные системы можно классифицировать по объекту исследования, степени инвазивности, методам спектральной селекции, спектральному диапа- зону и алгоритмам обработки гиперспектрального куба данных [1].

Одной из задач, в которых может найти применение гиперспектральный анализ, является исследование повреждений и заболеваний кожи, в том числе онкологических [1-3]. Для решения этой задачи гиперспектральные системы могут работать как совместно с эндоскопами для исследования биологических тканей in vivo [4], так и в составе микроскопов для исследований in vivo [5,6] и in vitro [7]. Оба подхода могут дополнить существующие методы диагностики рака, прежде всего, морфологическое и иммуногистохимическое исследование, флуоресцентную спектроскопию [8], лазерную автофлуоресцентную поляриметрию [9], спектроскопию комбинационного рассеяния [10].

Другое весьма важное применение гиперспектрального анализа - цифровая голографическая микроскопия. Исследования $[11,12]$ показывают, что раковые клетки обладают более высоким показателем преломления по сравнению с нормальными клетками. Однако в известных на сегодняшний момент установках измерение пока- 
зателя преломления ведется либо в монохроматическом, либо в белом свете, в то время как дополнительное исследование присущей веществам дисперсии за счет регистрации цифровых голограмм в узких спектральных каналах в широком диапазоне спектра позволило бы повысить информативность и точность диагностики, а также выявить новые закономерности за счет детального анализа молекулярного состава вещества $[13,14]$.

Для использования в качестве устройств спектральной селекции интерес представляют акустооптические (АО) перестраиваемые фильтры. К их достоинствам относится программное управление, отсутствие подвижных элементов, высокое (до $0.1 \mathrm{~nm}$ ) спектральное разрешение и возможность создания гибких алгоритмов гиперспектрального анализа благодаря произвольной спектральной адресации в пределах рабочего интервала длин волн.

Гиперспектральным системам, в том числе на основе АО фильтров (АОФ), работающим совместно с микроскопами, присуще небольшое поле зрения [1], что требует применения алгоритмов объединения множества кадров и приводит к возникновению артефактов и снижению качества изображения. Более того, ранее было показано [15], что использование АОФ совместно с микроскопом без дополнительной сопрягающей оптической системы (ОС) приводит к существенному уменьшению поля зрения.

Данная работа посвящена созданию гиперспектрального модуля на основе АОФ, работающего с микроскопами, в котором за счет использования специально рассчитанной сопрягающей ОС сохраняется поле зрения микроскопа, минимизируется виньетирование и светоэнергетические потери.

\section{Схемы АО видеоспектрометров для исследования микрообъектов}

При решении многих задач анализа микрообъектов необходимо осуществлять спектральную фильтрацию излучения широкополосного источника. Для этого устройства спектральной селекции, в том числе АОФ, могут быть установлены как в осветительном, так и в одном из регистрирующих каналов микроскопа. Например, в работе [16] АОФ устанавливается в дополнительном съемном осветительном модуле и используется для выбора длины волны возбуждения флуоресценции из диапазона излучения широкополосного лазера (рис. $1, a)$.

В том случае, когда для гиперспектрального анализа необходимо использовать встроенную в микроскоп осветительную систему, состоящую из источника света и системы линз, размещение АОФ непосредственно в осветительном канале становится проблематичным ввиду ограниченности пространства осветительных систем. В этом случае целесообразно устанавливать АОФ не в осветительном, а в приемном канале.
Известны видеоспектрометры, в которых АОФ располагается в одном из стандартных приемных каналов ОС микроскопа - цифровом (рис. $1, b$ ) и визуальном (рис. 1,c). В работах, посвященных данным видеоспектрометрам, либо совсем не рассматривается вопрос эффективного сопряжения ОС АОФ с предшествующей ОС микроскопа [17], либо решается задача сопряжения АОФ с окуляром прибора [18]. Размещение АОФ за окуляром микроскопа ведет к частичной или полной потере визуального канала наблюдения и увеличению габаритов прибора, что не всегда приемлемо и конструктивно реализуемо.

Поэтому в данной работе предлагается установить АОФ таким образом, чтобы сохранить имеющиеся в микроскопе широкополосные каналы наблюдения и регистрации (визуальный и цифровой), дополнив их еще одним - спектральным (рис. 1,d). Размещение АОФ в отдельном съемном блоке - гиперспектральном модуле - между станиной микроскопа и тринокулярной насадкой обеспечивает универсальность, портативность и простоту встраивания модуля в микроскопы. Пример реализации такого подхода представлен на рис. 2.

Конструктивно АОФ может представлять собой одну или две ориентированные определенным образом и расположенные последовательно АО ячейки [19], в которых распространяется ультразвуковая волна и происходит дифракция света. Двойная монохроматизация излучения позволяет уменьшить пространственно-спектральные искажения, вносимые фильтром, за счет специального расположения одной $\mathrm{AO}$ ячейки относительно другой, а также повысить спектральное разрешение и спектральный контраст. В данной работе расчет проводился для двойного АОФ, работающего в спектральном диапазоне 450-750 nm и имеющего полосу пропускания 0.7-1.9 nm. Таким образом, с помощью него можно реализовать гиперспектральный анализ практически во всем видимом диапазоне с числом спектральных каналов $\sim 230$.

\section{Расчет сопрягающей оптической системы}

Для эффективного использования АОФ должно быть обеспечено его сопряжение с ОС микроскопа по светоэнергетическим параметрам и полю зрения. Следует отметить, что решение этой задачи крайне важно для получения качественных и информативных изображений, особенно когда их регистрация ведется в условиях низкой освещенности и на больших увеличениях. Проблема заключается в том, что АОФ имеют небольшую угловую апертуру $\left(2 \omega_{A O F} \sim 3^{\circ}\right)$, которая, как правило, меньше полного углового поля после микрообъектива микроскопа $\left(2 \omega_{m} \sim 5.7-8.9^{\circ}\right)[15]$, то есть без использования специальной сопрягающей ОС может быть потеряно до $\sim 90 \%$ линейного поля зрения. 


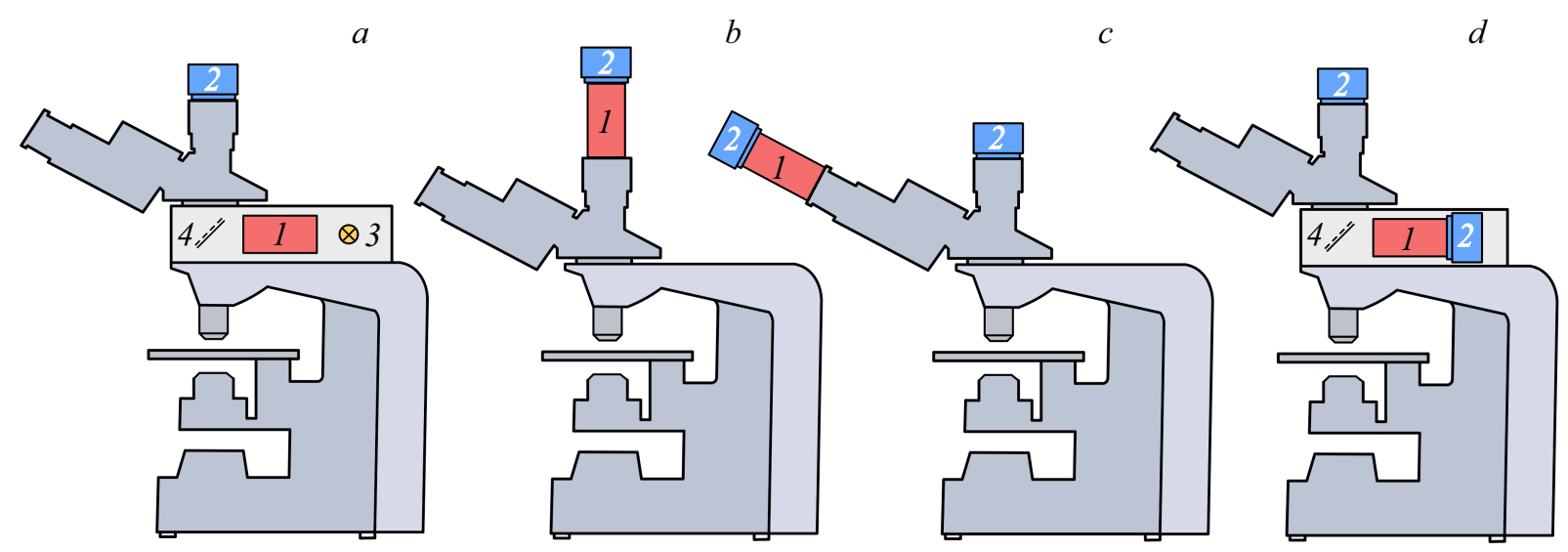

Рис. 1. Варианты расположения АОФ в микроскопе: в осветительном $(a)$ и приемных каналах: цифровом $(b)$, визуальном $(c)$ и спектральном $(d)$, выполненным в качестве отдельного модуля; 1 - АОФ, 2 - видеокамера, 3 - источник света, $4-$ светоделитель.

Сопрягающая ОС представляет собой афокальную систему, состоящую из двух положительных объективов и отрицательной линзы-коллектива, расположенной вблизи их сопряженной фокальной плоскости, в которой формируется промежуточное изображение. На рис. 3 представлен ход осевых и внеосевых лучей, выходящих из объектива микроскопа и поступающих в гиперспектральный модуль, где они преобразуются сопрягающей ОС, поступают в АОФ и фокусируются объективом камеры на приемник излучения.

Расчет проводился для микроскопа BW Optics MF-606 с угловым полем $2 \omega_{m} \approx 5.7^{\circ}$ и АОФ со следующими параметрами: угловое поле $2 \omega_{A O F}=3^{\circ}$, диаметр входного зрачка $D_{A O F}=7 \mathrm{~mm}$, длина фильтра $L=110 \mathrm{~mm}$. Соответствующая сопрягающая ОС должна: 1) преобразовывать угловое поле пучка на выходе микроскопа к угловому полю АОФ, $\omega_{O S}^{\prime}=\omega_{A O F}$, что выполняется при условии $\Gamma_{O S}=\operatorname{tg} \omega_{A O F} / \operatorname{tg} \omega_{m}=0.5 \times$, где $\Gamma_{O S}-$ увеличение сопрягающей ОС, и 2) обеспечивать минимальное виньетирование пучков, что достигается путем расположения выходного зрачка $P$ сопрягающей ОС внутри ОС АОФ, а также стремлением к равенству диаметра пучка на выходе сопрягающей ОС и диаметра входного зрачка $\mathrm{AO \Phi ,} D_{O S}^{\prime}=D_{A O F}$. Согласно инварианту Гельмгольца [20], связывающему линейный размер предмета и угловой размер пучка лучей, для сопрягающей ОС будет справедливо выражение $\omega_{m} D_{m}=\omega_{O S}^{\prime} D_{O S}^{\prime}$, где $D_{m}$ - диаметр пучка после объектива микроскопа в плоскости сопряжения с гиперспектральным модулем. С учетом указанного выше требования $\omega_{O S}^{\prime}=\omega_{A O F}$ последнее выражение преобразуется к $\omega_{m} D_{m}=\omega_{A O F} D_{O S}^{\prime}$. Поскольку $\omega_{A O F}<\omega_{m}$, то $D_{O S}^{\prime}>D_{m} \approx 30 \mathrm{~mm}$, а значит $D_{O S}^{\prime}>D_{A O F}=7 \mathrm{~mm}$. Таким образом, преобразование углового поля к меньшему значению приводит к увеличению диаметра пучка, который превосходит диаметр входного зрачка АОФ, что ведет к светоэнергетическим потерям. Однако ввиду того, что задача минимизации светоэнергетических потерь может быть частично реше-

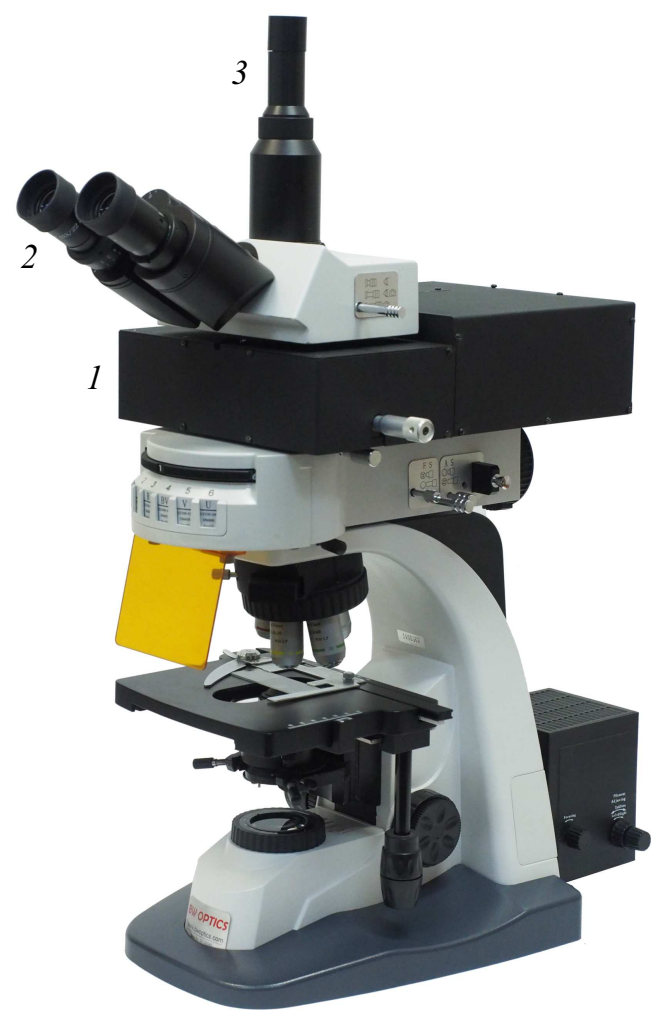

Рис. 2. АО видеоспектрометр на основе микроскопа: 1 - гиперспектральный модуль; 2 - визуальный канал наблюдения; 3 - цифровой канал регистрации изображений в белом свете.

на путем выбора более мощного источника излучения и увеличения времени экспонирования, в ходе расчета приоритет был отдан сохранению поля зрения, то есть выполнены соотношения $\omega_{O S}^{\prime}=\omega_{A O F}, D_{O S}^{\prime}>D_{A O F}$.

Таким образом, в рассчитанной ОС неизбежно присутствует виньетирование пучков. Для его количественного описания, а также в качестве критерия выбора той или иной конструкции сопрягающей ОС предла- 


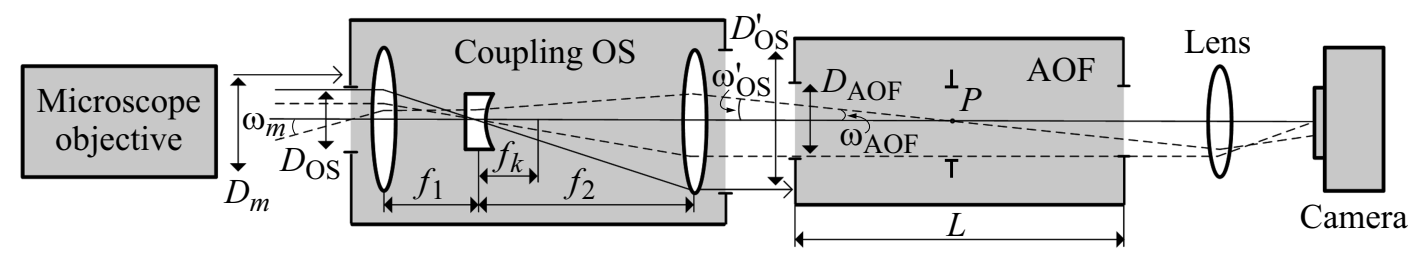

Рис. 3. Оптическая схема гиперспектрального модуля.
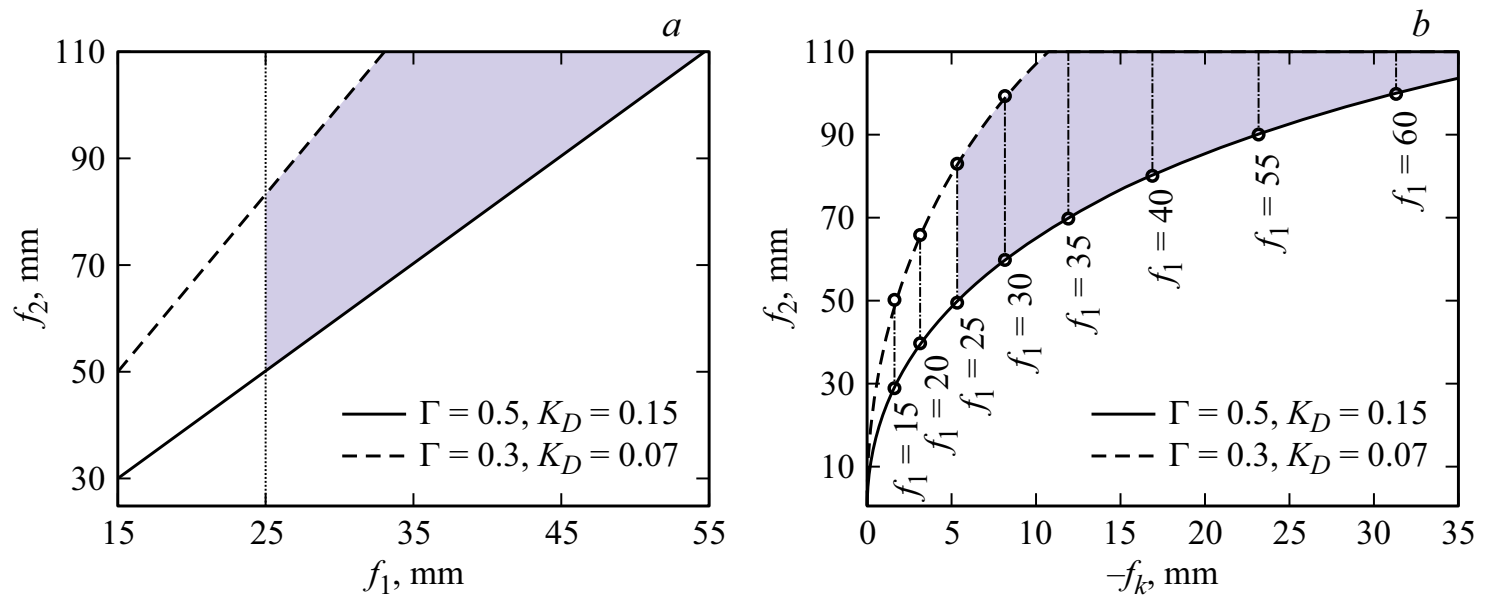

Рис. 4. Диапазон фокусных расстояний элементов сопрягающей ОС $(a)$ и зависимость значений фокусного расстояния коллективной линзы от значений фокусного расстояния первого элемента сопрягающей ОС $(b)$.

гается использовать энергетический коэффициент сопряжения [21]: $K_{D}=\left(D_{A O F} / D_{O S}^{\prime}\right)^{2}$, значение которого тем выше, чем меньше виньетирование. Для минимизации виньетирования используют коллективную линзу, значение фокусного расстояния $f_{k}$ которой влияет на положение выходного зрачка $P$ афокальной ОC [22]. Значение $f_{k}$ подбирают таким образом, чтобы наибольшая часть внеосевых пучков после сопрягающей ОС поступила в АОФ. Фокусное расстояние коллективной линзы определялось в результате операции оптимизации в программе Zemax. Критерием оптимизации служило расположение выходного зрачка $P$ сопрягающей ОС на расстоянии $L / 2=55 \mathrm{~mm}$ от входного окна АОФ. Элементы афокальной ОС в ходе расчета были представлены в виде тонких компонентов.

Для преобразования углового поля микроскопа к угловому полю АОФ в зависимости от модели микроскопа должно быть выполнено условие $\Gamma_{O S}=\operatorname{tg} \omega_{A O F} / \operatorname{tg} \omega_{m}=$ $=3 /(5.7-8.9)=(0.3-0.5) \times$. На рис. $4, a$ представлен график, показывающий область возможных значений фокусных расстояний $f_{1}$ и $f_{2}$ объективов сопрягающей OC, соответствующую рассчитанному диапазону увеличений $\Gamma_{O S}$. Диапазон значений $f_{1}$ ограничен снизу ввиду слишком малых значений фокусных расстояний $f_{k}$ коллективной линзы. Из рис. $4, b$ видно, что при $f_{1}<25 \mathrm{~mm}$ расчет дает решения для $f_{k}$, требующие большой кривизны поверхностей отрицательной линзы. Сверху значения $f_{1}$ и $f_{2}$ могут быть ограничены тре- бованием компактности сопрягающей ОС. Из рис. 4, $b$ также следует, что значения $f_{k}$ слабо зависят от $f_{2}$ и полностью определяются первым элементом сопрягающей OC.

Для определения энергетического коэффициента сопряжения $K_{D}$ световые диаметры рассчитывались исходя из заданной числовой апертуры $N A=0.65$ в плоскости предметов, определяемой микрообъективом с увеличением $\beta=40 \times$, который наиболее часто используется для исследования гистологических срезов. Расчет показывает, что значение $K_{D}$ остается постоянным для данного увеличения ОС независимо от сочетания фокусных расстояний $f_{1}$ и $f_{2}$ (рис. $\left.4, a, b\right)$. Это позволяет из множества вариантов выделить те, которые обеспечивают малогабаритную конструкцию сопрягающей ОС. При этом следует отметить, что варианты афокальной ОС с $f_{1} \leq D_{m} \approx 30 \mathrm{~mm}$ малоэффективны, поскольку в этом случае даже при использовании светосильного первого объектива сопрягающей ОС с диафрагменным числом $k=2$ его световой диаметр $D_{O S}$ окажется вполовину меньше диаметра пучка $D_{m}$ после микрообъектива в плоскости присоединения модуля (рис. 3), что приведет к существенным светоэнергетическим потерям.

C учетом вышеизложенного установлено, что оптимальная по технологическим, габаритным, светоэнергетическим и полевым характеристикам конструкция сопрягающей ОС для гиперспектрального модуля будет удовлетворять сочетаниям $f_{1}=35 \mathrm{~mm}$, 

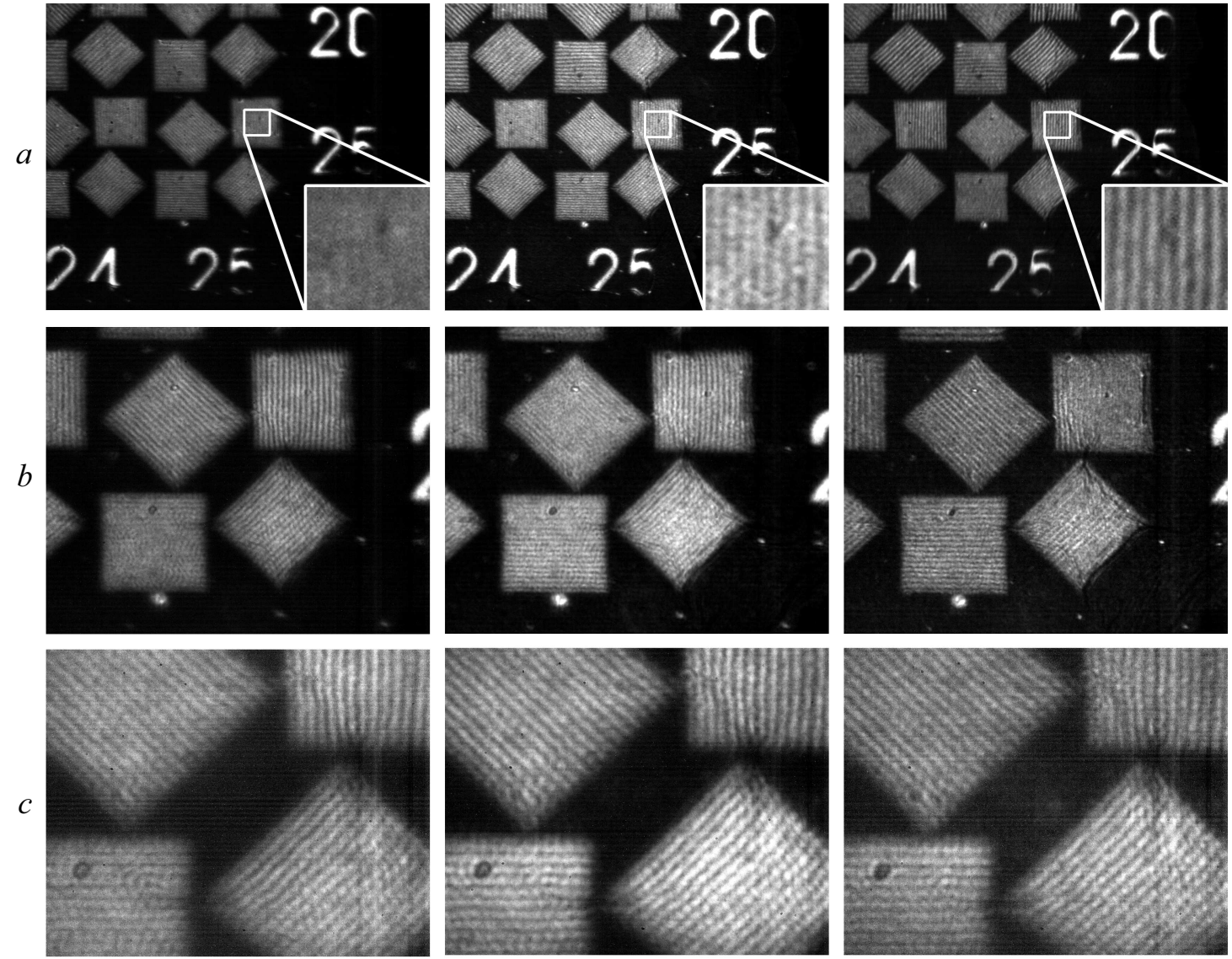

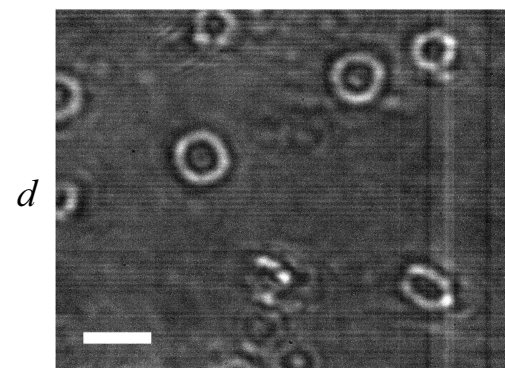

$550 \mathrm{~nm}$

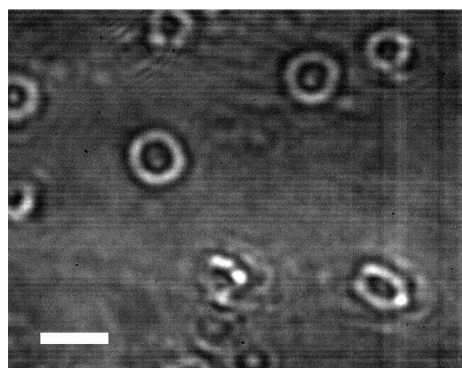

$640 \mathrm{~nm}$

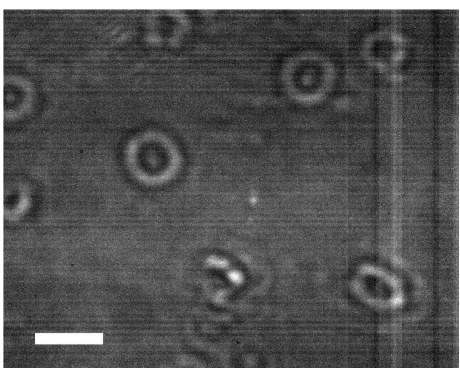

$730 \mathrm{~nm}$

Рис. 5. Спектральные изображения штриховой миры с увеличением микрообъектива $10 \times(a), 20 \times(b), 40 \times(c)$ и эритроцитов крови человека с увеличением $100 \times$ (длина масштабного отрезка $10 \mu \mathrm{m})(d)$, полученные с помощью видеоспектрометра.

$f_{2}=70-100 \mathrm{~mm}, f_{k}=-12 \mathrm{~mm}$. Второй элемент афокальной ОС может быть выполнен в виде объектива с фиксированным фокусным расстоянием для определенного значения углового поля конкретной модели микроскопа, либо может представлять собой вариофокальный объектив, обеспечивая, таким образом, совместимость с любыми моделями микроскопов.

Следует отметить сложность задачи непосредственного расчета аберраций в приборах с АОФ, связанную с их пространственно-спектральной зависимостью [23]. Поэтому для их полноценной компенсации необходимо проводить калибровку и пространственную коррекцию изображений для каждого прибора по известной методике, например, представленной в работе [24].

\section{Экспериментальные результаты}

Рассчитанная в данной работе сопрягающая ОС была реализована на основе двух объективов с фокусными расстояниями $f_{1}=35 \mathrm{~mm}$ и $f_{2}=70 \mathrm{~mm}$. Между объективами был установлен отрицательный коллектив, представляющий собой плосковогнутую линзу с фокусным расстоянием $f_{k}=-12 \mathrm{~mm}$, который был выбран в соответствии с графиком на рис. $4, b$. После сопрягающей ОC был установлен АОФ и затем объектив с переменным фокусным расстоянием $100-210 \mathrm{~mm}$ для возможности регулировки поля зрения, регистрируемого приемником излучения. В данной работе была использована камера DMK 23UX236 (The Imaging Source) с сенсором 1/2.8", обладающим разрешением $1920 \times 1080$ элементов. В ка- 


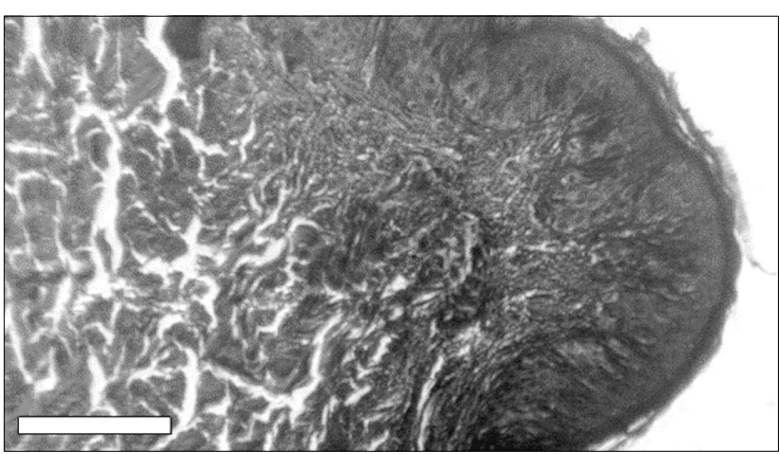

$530 \mathrm{~nm}$

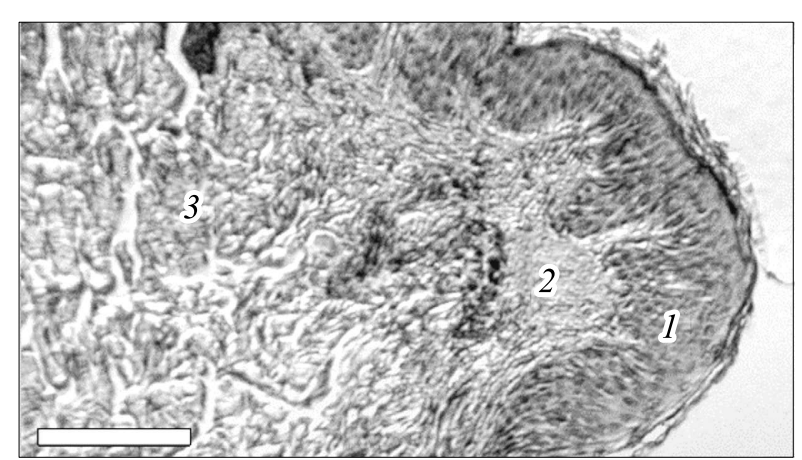

$630 \mathrm{~nm}$

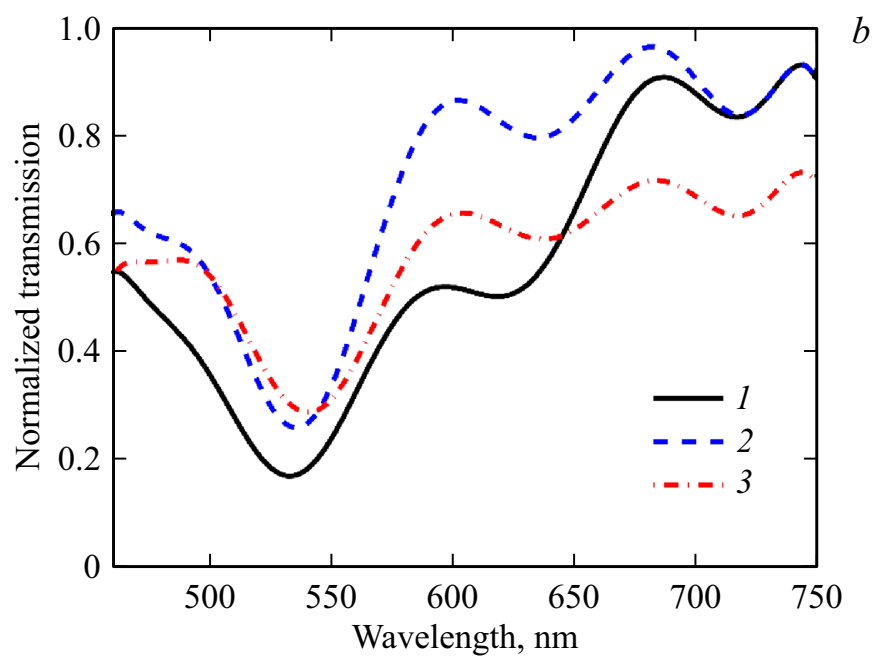

Рис. 6. (a) Изображения микропрепаратов кожи на длине волны 530 и $630 \mathrm{~nm}$ (длина масштабного отрезка $100 \mu$ m, увеличение микрообъектива $10 \times) ; 1$ - эпидермис, 2 - сосочковый и 3 - сетчатый слои дермы; $(b)$ нормированные спектральные кривые пропускания тканей кожи, обозначенных цифрами на рис. $a$.

честве широкополосного источника излучения использовалась галогенная лампа мощностью $100 \mathrm{~W}$.

Для оценки предела разрешения гиперспектрального модуля в составе микроскопа были зарегистрированы спектральные изображения 25-го элемента штриховой миры № 1 [25] и эритроцитов в мазке крови человека (рис. 5). Каждый элемент миры состоит из четырех групп параллельных штрихов, равных по ширине и ориентированных в различных направлениях. Ширина штриха и расстояние между соседними штрихами равны, и для 25-го элемента миры № 1 период штрихов составляет $5 \mu \mathrm{m}$, что соответствует разрешающей способности 200 lines $/ \mathrm{mm}$. Значения разрешающей способности для микрообъективов различных увеличений $\beta$ представлены в таблице. Видно, что при использовании микрообъективов с увеличением $20 \times$ и $40 \times$ видеоспектрометр способен разрешить наименьший элемент миры, однако в случае объектива с увеличением $10 \times$ разрешающая способность видеоспектрометра уменьшается до 180-190 lines/mm для некоторых групп штрихового элемента, имеющих вертикальное и горизонтальное направление линий в коротковолновой и длинноволновой
Разрешающая способность видеоспектрометра, $\mathrm{mm}^{-1}$, не менее

\begin{tabular}{c|c|c|c|c|c}
\hline \multirow{2}{*}{$\beta, \times$} & \multicolumn{4}{|c}{$\lambda, \mathrm{nm}$} \\
\cline { 2 - 6 } & \multicolumn{2}{|c|}{550} & 640 & \multicolumn{2}{c}{730} \\
\hline \multirow{2}{*}{10} & 200 & 190 & \multirow{2}{*}{200} & 200 & 200 \\
\cline { 2 - 3 } \cline { 2 - 5 } & 200 & 200 & & 180 & 200 \\
\hline 20 & \multicolumn{2}{|c|}{200} & 200 & \multicolumn{2}{|c}{200} \\
\hline 40 & \multicolumn{2}{|c|}{200} & 200 & \multicolumn{2}{|c}{200}
\end{tabular}

области спектрального диапазона АОФ, соответственно. Это может быть обусловлено различным характером коррекции астигматизма оптической системы видеоспектрометра в целом на этих длинах волн.

Снимки эритроцитов, полученные при увеличении микрообъектива $100 \times$, демонстрируют достаточно хорошее качество изображения и сохранение поля зрения микроскопа с помощью рассчитанной сопрягающей ОС. 
Диагональ зарегистрированных кадров при этом увеличении составляет $85 \mu \mathrm{m}$.

Перед исследованием биообразцов была проведена калибровка видеоспектрометра по данным регистрации спектральных изображений калибровочной сетки [24]. Все последующие изображения подвергались пространственной коррекции согласно данным калибровки. Для исключения влияния на гиперспектральный куб данных различных факторов (неравномерность освещенности поля зрения, спектральная зависимость излучения источника света, спектральные характеристики пропускания оптических элементов и пр.) был проведен предварительный гиперспектральный анализ фона в отсутствие образца. С учетом этого далее зарегистрированные изображения микропрепаратов корректировались, в результате чего были получены нормированные спектральные характеристики пропускания образцов.

Для демонстрации возможностей гиперспектрального модуля были зарегистрированы спектральные изображения поперечного среза кожи человека, окрашенного гематоксилин-эозином (рис. 6, $a$ ), и получены нормированные спектральные характеристики пропускания различных тканей кожи: эпидермиса, сосочкового и сетчатого слоев дермы (рис. 6, $b$ ). Как было показано в работе [26], изучение спектров окрашенных гематоксилинэозином препаратов позволяет достоверно различать области здоровой кожи, доброкачественных образований и меланомы при условии стандартизированной подготовки образцов, включающей заданное время окраски и толщину среза. Разработанный гиперспектральный модуль может быть использован для решения этой и других диагностических задач.

\section{Заключение}

В работе проведен расчет и анализ сопрягающей ОС, которая позволила эффективно встроить АО фильтр в световой микроскоп для осуществления гиперспектрального анализа биологических микрообъектов и расширить функциональные возможности микроскопов для диагностики различных заболеваний, в том числе онкологических. Рассчитаны значения фокусных расстояний $f_{1}, f_{k}$ и $f_{2}$ элементов сопрягающей ОС и даны рекомендации для их оптимального выбора, что позволяет оптимизировать параметры системы для конкретной модели микроскопа. Рассчитанная ОС позволяет избежать потери части поля зрения, которая в ее отсутствие могла бы составлять до $90 \%$.

Достоинством предлагаемого подхода является обеспечение мультимодальности прибора, то есть возможности реализации одновременно нескольких режимов анализа: световой (в белом свете), спектральной (в режиме видеоспектрометра) и гиперспектральной цифровой голографической микроскопии. Режим гиперспектральной цифровой голографической микроскопии можно реализовать с помощью модификации предложенного модуля, заменив объектив, следующий за АОФ, на компактный интерферометр общего пути [13]. При этом приведенный расчет системы сопряжения останется справедлив и для данной модификации.

При реализации такого подхода появляется возможность исследования спектральной зависимости показателя преломления тканей и клеток, что, как и данные гиперспектрального анализа, может быть использовано для диагностики широкого круга заболеваний, включая заболевания кожи различной этиологии.

Работа выполнена при поддержке грантов РФФИ № 17-38-50145 и 18-29-02108. Результаты работы получены с использованием оборудования Центра коллективного пользования Научно-технологического центра уникального приборостроения РАН.

\section{Список литературы}

[1] Lu G., Fei B. // J. Biomed. Opt. 2014. V. 19(1). P. 010901. doi 10.1117/1.JBO.19.1.010901

[2] Братченко И.А., Алонова М.В., Мякинин О.О., Морятов А.А., Козлов С.В., Захаров В.П. // Компьютерная оптика. 2016. Т. 40. № 2. С. 240. doi 10.18287/2412-61792016-40-2-240-248

[3] Diebele I., Kuzmina I., Lihachev A., Kapostinsh J., Derjabo A., Valeine L., Spigulis J. // Biomedical Optics Express. 2012. V. 3. N 3. P. 467. doi 10.1364/BOE.3.000467

[4] Kong S.G., Martin M.E., Vo-Dinh T. // Anglais. 2006. V. 28. N 6. P. 770. doi 10.4218/etrij.06.0106.0061

[5] Sorg B.S., Moeller B.J., Donovan O., Cao Y., Dewhirst M.W. // J. Biomed. Opt. 2005. V. 10. N 4. P. 44004. doi 10.1117/1.2003369

[6] Palmer G.M., Fontanella A.N., Zhang G., Hanna G., Fraser C.L., Dewhirst M.W. // J. Biomed. Opt. 2010. V. 15. N 6. P. 066021. doi $10.1117 / 1.3523363$

[7] Shah S.A., Bachrach N., Spear S.J., Letbetter D.S., Stone R.A., Dhir R., Prichard J.W., Brown H.G., LaFramboise W.A. // Biotechniques. 2003. V. 34. N 2. P. 408.

[8] Borisova E., Zhelyazkova Al., Keremedchiev M., Penkov N., Semyachkina-Glushkovskaya O., Avramov L. // Optics and Spectroscopy. 2016. T. 120. № 1. C. 38. doi 10.7868/S0030403416010062

[9] Ушенко Ю.А. // Optics and Spectroscopy. 2016. Т. 118. № 6. C. 1040. doi 10.7868/S0030403415060239

[10] Zakharov V.P., Bratchenko I.A., Artemyev D.N., Myakinin O.O., Khristoforova Y.A., Kozlov S.V., Moryatov A.A. // Proc. SPIE. 2015. V. 9537. P. 95372H. doi $10.1117 / 12.2186095$

[11] Choi W.J., Jeon D.I., Ahn S.G., Yoon J.H., Kim S., Lee B.H. // Opt. Express. 2010. V. 18. N 22. P. 23285. doi 10.1364/OE.18.023285

[12] Wang Z., Tangella K., Balla A., Popescu G. // J. Biomed. Opt. 2011. V. 16. N 11. P. 116017. doi 10.1117/1.3656732

[13] Mir M., Bhaduri B., Wang R., Zhu R., Popescu G. // Progress in Optics. 2012. V. 57. P. 133-217. doi 10.1016/B978-0-44459422-8.00003-5

[14] Machikhin A.S., Polschikova O.V., Ramazanova A.G., Pozhar V.E. // Journal of Optics. 2017. V. 19. № 7. P. 075301. doi 10.1088/2040-8986/aa72a7 
[15] Polschikova O., Machikhin A., Batshev V., Ramazanova A., Belov A., Pozhar V. // Proc. SPIE. 2017. V. 10592. P. 105920H. doi $10.1117 / 12.2297614$

[16] Shepard K.L., Field R.M. Патент US9831283B2, 2017.

[17] Kutuza I.B., Pozhar V.E., Pustovoit V.I. // Proc. SPIE. 2003. V. 5143. P. 165 . doi $10.1117 / 12.500528$

[18] Machikhin A., Batshev V. // Instruments and Experimental Techniques. 2014. V. 57. N 6. P. 736. doi $10.1134 / \mathrm{S} 0020441214060086$

[19] Mazur M.M., Shorin V.N., Abramov A.Yu., Magometov Z.A., Mazur L.I. // Opt. Spectrosc. 1996. V. 81. P. 475.

[20] Bass M., editor-in-chief. Handbook of Optics. Vol. I. Geometrical and Physical Optics, Polarized Light, Components and Instruments. McGraw-Hill, 2010. $1251 \mathrm{P}$.

[21] Мачихин А.С., Батшев В.И., Перчик А.В. // Труды Оптического общества им. Д.С. Рождественского. 10 Международная конференция „Прикладная оптика-2010“. 16-19 окт. 2012 г., $132-135$.

[22] Handbook of optics. Vol. II. Devices, Measurements, and Properties. Ed. by Bass M., McGraw-Hill, 1995. 1496 P.

[23] Pozhar V., Pustovoit V. // Photonics and optoelectronics. 1997. V. 4. № 2. P. 67.

[24] Machikhin A.S., Shurygin A.V., Pozhar V.E. // Instruments and Experimental Techniques. 2016. V. 59. N 5. P. 692. doi 10.1134/S0020441216040217.

[25] ГОСТ 15114-78. Системы телескопические для оптических приборов. Визуальный метод определения предела разрешения.

[26] Dicker D.T., Lerner J., Belle P.V., Barth S.F., Guerry D., Herlyn M., Elder D.E., El-Deiry W.S. // Cancer Biol. Ther. 2006. V. 5. N 8. P. 1033. doi 10.4161/cbt.5.8.3261. 